\title{
Spectrum of Interface Dermatitis : An Observational Study
}

\author{
Vishrabdha Rahul Pawar ${ }^{1}$, Vaibhav Pandurang Mane ${ }^{1 *}$ and Renuka Satish Ashtekar ${ }^{2}$ \\ 'Department of Pathology, Bharati Vidyapeeth Deemed University Medical College And Hospital, Sangli. India \\ ${ }^{2}$ Department of Dermatology and Skin VD, Bharati Vidyapeeth Deemed University Medical College And Hospital, Sangli, India
}

\begin{abstract}
Background: Major role of a dermatopathologists is to try to make specific diagnosis of inflammatory skin diseases. IFD means inflammatory infiltrate that abuts or obscures the dermo-epidermal junction in askin biopsy.This type of reaction is also seen in skin disorders associated with systemic illness like LE and skin changes of potentially fatal disorders such as Graft versus host disease, S-J syndrome and toxic epidermal necrolysis.

Aims \& Objectives: 1)To study the histopathology of interface dermatitis.(2)To study the common types of skin manifestations in interface dermatitis.(3)To determine other histological features associated with interface dermatitis.4)To identify the clinical concordance of various types of interface dermatitis.

Material \& Methods: Present study is a retrospective , Hospital based study. Ethical consideration was taken. Study duration was Jan 2012 to Dec 2015 .

Results: In the present study, a total of 121 cases of IFD were studied, the majority of which [65] presented as papulo- squamous [ papules and plaques ] lesions. Of theses 121 cases, the most common type of IFD was LP and its variants (53.71\%). The next common was LDE $(8.26 \%)$ followed by PL(7.4\% ). The least common was LN $(0.8 \%)$ and dermatomyositis (1.6 \%). Female preponderance was noted in case of IFD [ $59.9 \%$ ]. The percentage of clinical concordance was good in cases of LP \& its variants (90.77 \%), DLE ( $83.33 \%)$ and in Dermatomyositis.
\end{abstract}

Conclusion: A good clinicopathologic correlation is absolutely essential for the final diagnosis of IFD.

Keywords: Interface, Dermatitis, Lichen

\section{Introduction}

LTR or IFD is one of the common clinical and histological presentation in Dermatology and Pathology. IFD refers to the finding of an inflammatory infiltrate that abuts or obscures the dermo-epidermal junction in a skin biopsy ${ }^{[1]}$ Interface reactions are so named because they are cell mediated immunologic reactions where the basal keratinocytes that reside above the dermo-epidermal junction are the target. ${ }^{[2]}$ One of the challenging aspects of being a dermatopathologists is to try to make specific diagnosis of inflammatory skin diseases. Now the microscopic findings of many inflammatory skin diseases and the most expert dermatopathologists are able to handle discrepancies between clinical and histopathological findings. This has changed the negative feelings of clinical dermatologists regarding the utility of biopsy in diagnosis of inflammatory conditions ${ }^{[3]}$

Lichen Planus is the prototype of the term "Lichenoid' which refers to papular lesion of certain skin disorders. ${ }^{[4]}$. This type of reaction can also be seen in skin disorders associated with systemic illness like LE and skin changes of potentially fatal disorders such as Graft versus host disease, S-J syndrome and toxic epidermal necrolysis ${ }^{[5]}$

\section{Components of the infiltrate in IFD are -}

i. Basal layer of epidermis having tall columnar cells perpendicular to basement membrane.

ii. Dermo-epidermal junction.

iii. The papillary dermis in contact with basement membrane.

iv. The adventitial dermis around the adnexal structures.

\section{Le Boit PE has classified ID into 5 types :}

i. Acute cytotoxic type

ii. IFD with premature terminal differentiation

iii. IFD with irregular epidermal hyperplasia

iv. IFD with psoriasiform hyperplasia.

v. IFD with epidermal atrophy.

\section{Aims and Objectives}

- To study the histopathology of interface dermatitis

- To study the common types of skin manifestations in interface dermatitis.

- To determine other histological features associated with interface dermatitis. 
- To identify the clinical concordance of various types of interface dermatitis.

\section{Material \& Methods}

Present study is a retrospective study, done in a tertiary care hospital. Ethical consideration was taken. Study duration was Jan 2012 to Dec 2015. The skin biopsy samples received in department of Pathology in this period were studied. We retrieved 402 cases of skin biopsies received in this period, of which 121 showed the findings of IFD.

Inclusion Criteria: The skin biopsies showing the dermoepidermal inflammatory infiltrate.

Exclusion Criteria: All the skin biopsies showing the histopathological findings other than dermoepidermal inflammatory infiltrate.

Limitations: The sample size is less. Direct access to the patient to note down the demographic findings was not possibleThe demographic findings were collected from the history and clinical findings noted on the requisition forms procured from the record section.

Ethical consideration was taken.The studied data was analyzed by relevant statistical method. Limitation of this retrospective study was the small sample size and that there was no direct access to the patients, so we had to rely on the clinical details given in the requisition forms.

\section{Results}

In the present study, a total of 121 cases of IFD were studied, the majority of which [65 ] presented as papulosquamous [ papules and plaques ] lesions (53.71\%).
Of theses 121 cases, the most common type of IFD was LP and its variants $(53.71 \%)$. The next common was LDE $(8.26 \%)$ followed by PL $(7.4 \%)$. The least common was LN $(0.8 \%)$ and dermatomyositis $(1.6 \%)$.

IFD affects any age group. In the present study the majority cases were in the $4^{\text {th }}$ decade ( $\left.41-50 \mathrm{yrs}\right)(28.92 \%)$ followed by $31-40$ yr age group [19.83\%] and $51-60 \mathrm{yr}[18.18 \%]$.

Female preponderance was noted in case of IFD [59.9\%]. In all the cases of IFD studied the female preponderance was seen with majority cases of LP and its variants.

Clinical concordance was seen in 100[82.64\%] cases and discordance in $21[17.36 \%]$ cases. The percentage of clinical concordance was good in cases of LP \& its variants [90.77 \% ], DLE [ 83.33\% ] and in Dermatomyositis, we had only one case, [100\% ] due to characteristic clinical presentation.

\section{Discussion}

We have tabulated the results sequentially according to the frequency of cases that we have studied and we are following the histopathological classification of Le Boit. In this the epidermal changes are taken as classifying IFD into five groups ${ }^{[2]}$. In most of the cases the diagnosis was consistent and easy to observe, but in few cases clinical correlation was necessary.

In group I- Acute Cytotoxic type- Basal cell vacuolization with lymphocytic infiltrate in the lower epidermis with scattered necrotic keratinocytes at various levels in the epidermis is the characteristic finding. EM is the prototype of this category. Other conditions described with this

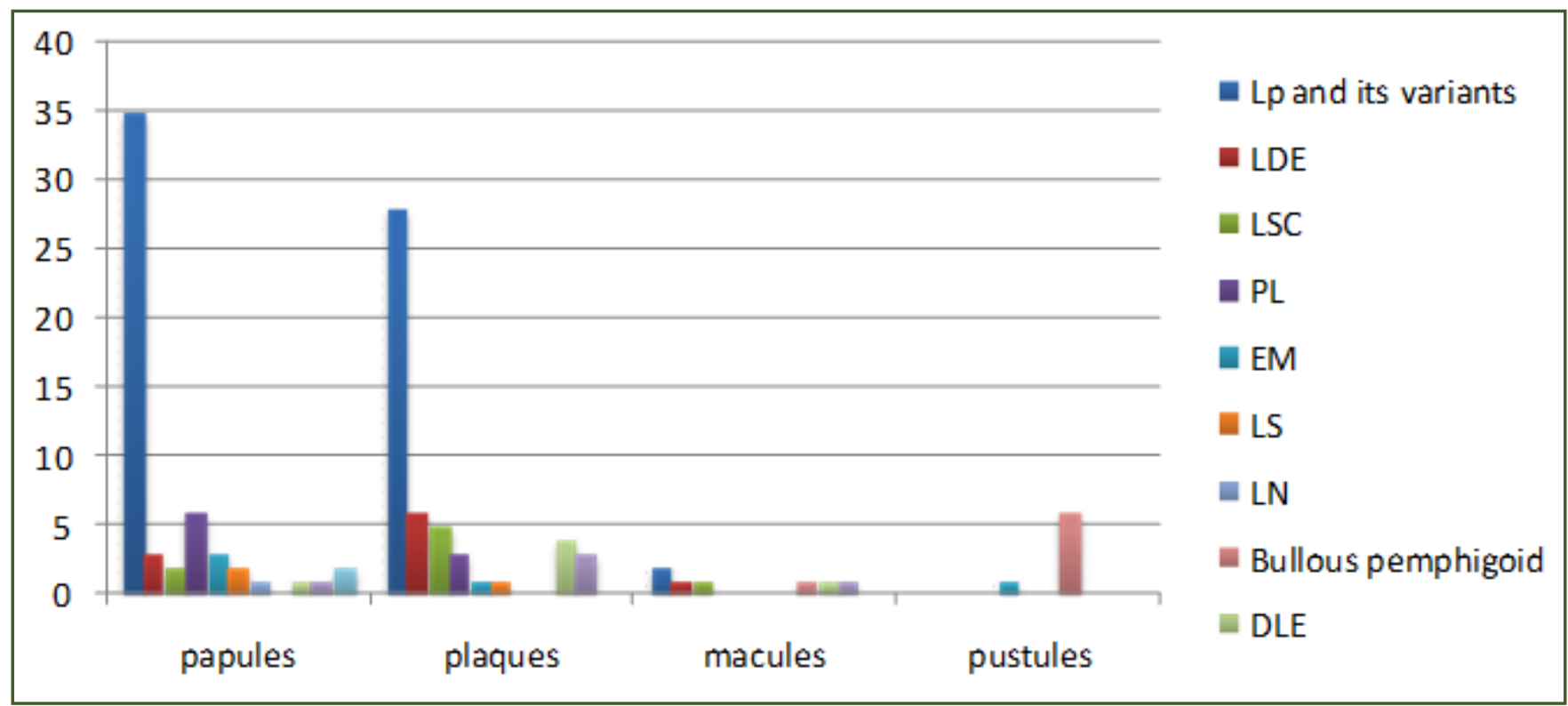

Table 1: The types of skin manifestations in ID. 


\section{No.of cases}

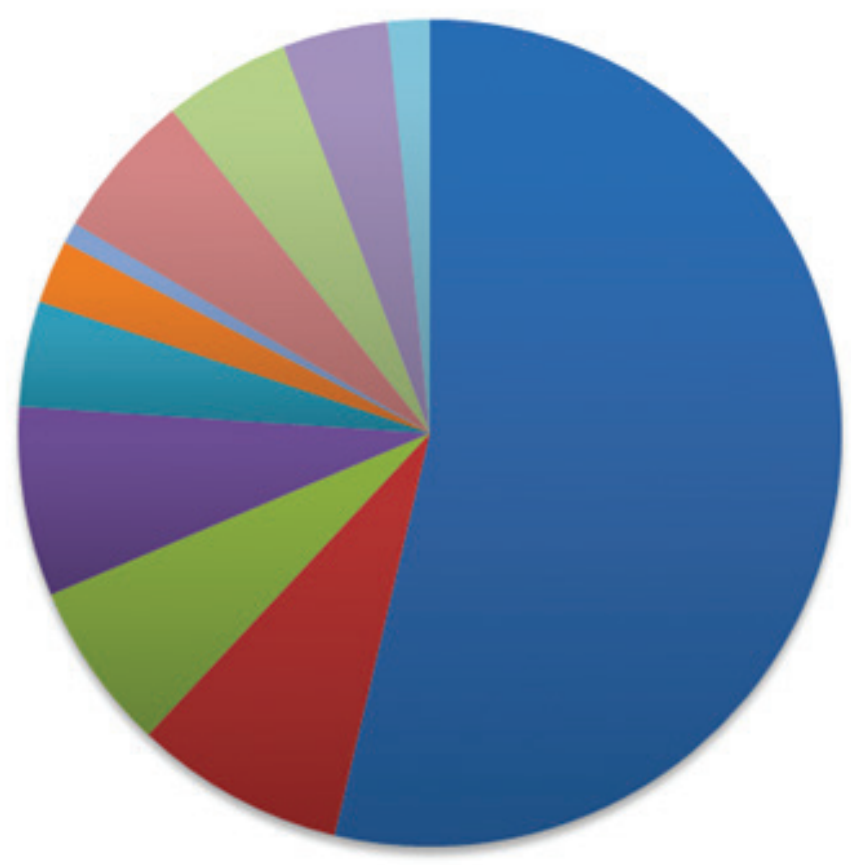

- LP \&its variants

ㄴ.DE

- LSC

口 PL

EM

ㄴ. LS

ㅁN

Bullous pemphigoid

D DLE

- SLE

dermatomyositis

Table 2: Types of Interface dermatitis.

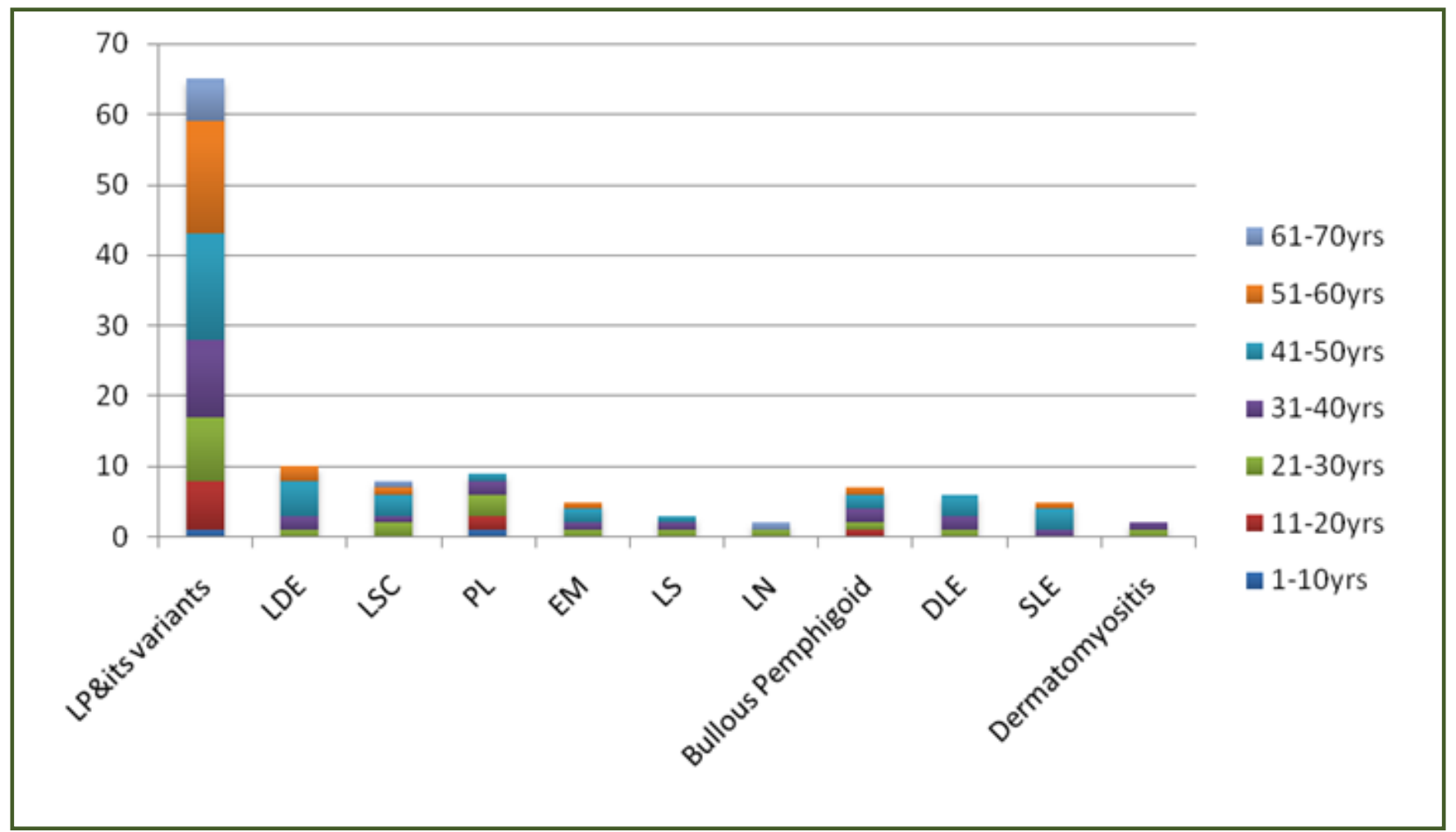

Table 3: Age distribution of lesion. 


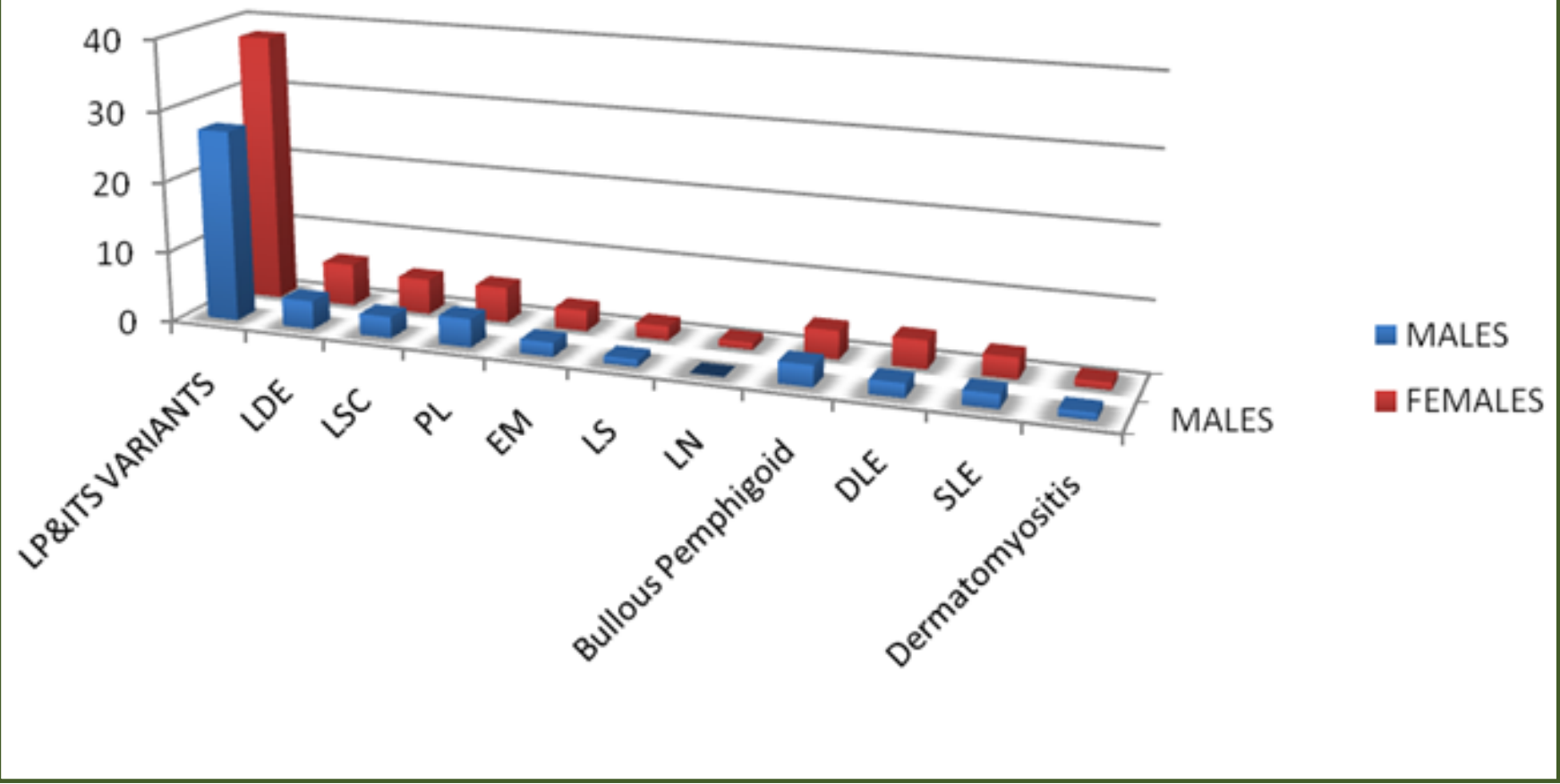

Table 4: Gender wise distribution of IFD.

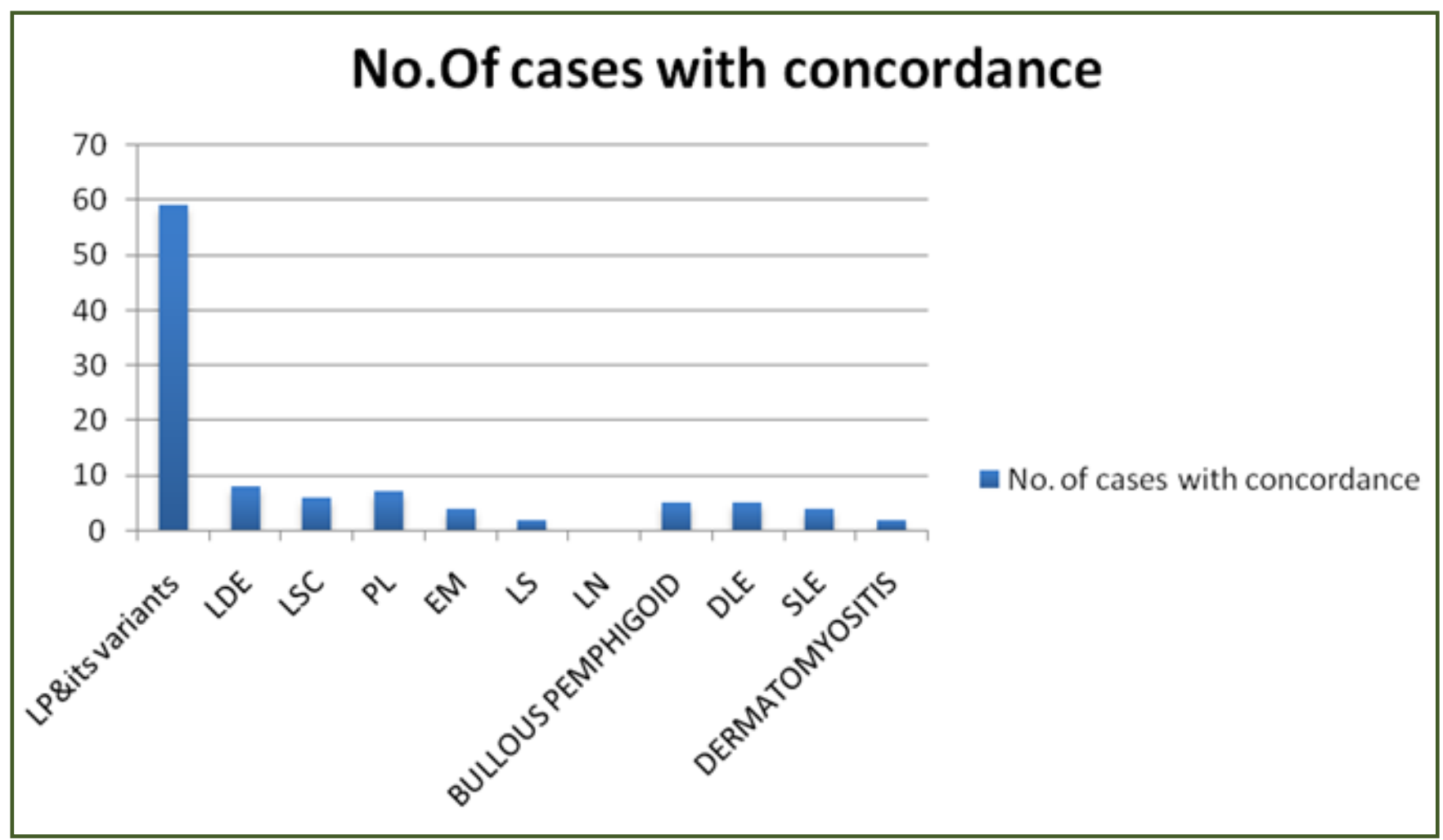

Table 5: Clinical concordance in IFD. 
Table 6: Frequency of different types of IFD as per Le Boit groups.

\begin{tabular}{|c|c|c|c|}
\hline Type of ID & Clinical condition & No. of cases & $\%$ \\
\hline \multirow{2}{*}{ I } & EM & 05 & 4.1 \\
\cline { 2 - 4 } & LDE & 10 & 8.26 \\
\hline \multirow{3}{*}{ II } & LP & 65 & 53.69 \\
\cline { 2 - 4 } & DLE & 06 & 4.9 \\
\cline { 2 - 4 } & LDE & 10 & 8.26 \\
\hline \multirow{2}{*}{ III } & Hypertrophic LP & 07 & 4.9 \\
\hline \multirow{2}{*}{$\vee$} & LS & 03 & 2.47 \\
\cline { 2 - 4 } & PLEVA & 09 & 3.4 \\
\hline \multirow{2}{*}{$V$} & Atrophic LP & 04 & 3.2 \\
\cline { 2 - 4 } & SLE & 05 & 4.13 \\
\cline { 2 - 4 } & DM & 02 & 1.6 \\
\hline
\end{tabular}

change are S-J syndrome, FDE, Acute Graft Versus Host reaction, PLEVA, TEN ${ }^{[2]}$. Vacuolar change is often an integral part of the basal cell damage in the lichenoid reaction. As a consequence of basal cell damage, there is variable melanin incontinence resulting from interference with melanin transfer from melanocytes to keratinocytes, which is associated with drug induced or solar induced lichenoid lesions ${ }^{[7]}$

In the present study we had 73(60\%) of 121 cases showing spongiosis and 25( $20.6 \%$ ) cases showed evidence of civatte bodies. Of these 05 cases were of EM (4.1\%), 09 cases $(7.4 \%)$ of PLEVA and 10 cases $(8.26 \%)$ cases of LDE. Sushma Desai et al ${ }^{[8]}$ studied 120 cases and found (6.6 \% )cases of EM. Suja Ajoy Kumar et al ${ }^{[9]}$ studied 71 cases and found $(5.6 \%) \mathrm{EM}$.

Erythema Multiforme-It is a clinical pathologic condition with a wide variety of underlying causes. The clinical finding has a targetoid morphology with a peripheral rim of erythema and a central zone of pallor. Some lesions manifest a dusky or violaceous appearance with no true central clearing. Blisters may be observed. As the pathogenetic basis of erythema multiforme is one of cellular cy-toxicity, the sites of predilection are those where anti-genic processing is maximal, which includes the palms and soles, but lesions may occur elsewhere and may become widespread ${ }^{[1]}$

In our study we studied 05 cases of EM with $80 \%$ clinical concordance. One case was missed clinically due to lack of classic clinical appearance. In 03 cases the blister formation was noted. Histopathologically 03 cases showed subepidermal bulla (Fig 2), dense lymphocytic tagging at the dermo-epidermal junction. Basket weave pattern of orthokeratosis and the characteristic basilar vacuolopathy is seen.(Fig 3). The melanin incontinence could be due to irritation. Civatte bodies are seen. (Figure 4)
Lichenoid Drug Eruptions- The lesion closely appears like LP clinically, there may be eczematization \& pronounced hyperpigmentation. LDE usually differs from LP by presence of focal parakeratosis and mild basal vacuolar change, few plasma cells \& eosinophils. There is more melanin incontinence than in LP. The infiltrate is often less and less band like than in LP itself:[7] A few inflammatory cells may extend around vessels in the mid and lower dermis. Sometimes the histology mimics LP, few eosinophils in the infiltrate may be the only clue diagnosis.

Pleva: Although both PLEVA and pityriasis lichenoides chronica (PLC) are considered to be variants of the same disease, namely, Mucha Haberman disease. The histological findings are different ${ }^{[6]}$ In our study we noted cases of PLEVA. (Fig 5) shows Hyperplastic epidermis and dense dermal infiltrate which is becoming Lichenoid. Also perivascular lymphocytic infiltration is noted. The upper dermis shows many extravasated RBCs.

In Group II - IFD with Premature Terminal Differentiation: Early development of a thick granular layer and compact stratum corneum which resembles acral skin and usually associated with dense lichenoid infiltrate of lymphocytes. LP is a prototype of this. Other conditions in which this pattern may be seen are DLE, LDE, GVHD, and Dermatomyositis, albeit with paucity of dermal infiltrate. In the present study we had $(69.4 \%)$ cases with hyperkeratosis and (66.9\%)cases of lymphocytic band like infiltrate in the dermis. We had $53.69 \%$ cases of LP, $(4.9 \%)$ of DLE, $(8.26 \%)$ of LDE and (1.6\%) of dermatomyositis. Suja Ajoy Kumar had $57.7 \%$ cases of LP, $15.4 \%$ cases of DLE and $1.4 \%$ cases of Dermatomyositis.

Lichen Planus- It is an idiopathic disorder.It affects skin and mucous membrane and is characterized by small, flat topped papules clinically and a band like mononuclear cell 
infiltrate histologically. The individual papules often have a polygonal shape, a violaceous to gray- red hue and a thin, retractile scale. Mucous membrane lesions have a lacy, white appearance ${ }^{[2]}$

In our study we found $53.69 \%$ cases of LP of which $4.9 \%$ were of Hypertrophic and $3.2 \%$ of atrophic variant. There was $90.77 \%$ clinical concordance in cases of LP. Histologically the epidermis was broad and acanthotic with saw-toothed pattern of rete pegs(Fig 6). Focal wedge shaped hypergranulosis is noted. The stratum corneum is moderately thick. The dense Lichenoid infiltrate of lymphocytes is seen occupying the widened papillary dermis. And is admixed with melanophages. Colloid bodies that represent anucleated remnants of apoptotic basal keratinocytes are seen.

DLE- We studied cases of DLE with $83.33 \%$ clinical concordance.(Fig 7) It shows Hyperkeratotic, acanthotic epidermis. Focal hypergranulosis is noted. Dense superficial and deep dermal lymphocytic infiltrate admixed with melanophages is noted. Perivascular infiltrate is shown in (Fig 8)

In Group III are Cases with Changes of Irregular Epidermal Hyperplasia. In this there is marked irregular epidermal hyperplasia as seen in Hypertrophic lichen planus, verrucous DLE and some long standing cases of LDE

In the present study we had $53.69 \%$ cases of LP , $4.9 \%$ cases of DLE and $8.26 \%$ cases of LDE with 7/121 cases of hypertrophic LP.

Sushma Desai et al had (4.1\%) of hypertrophic LP.

Suja Ajoy Kumar had single case of HTLP.

\section{Hypertrophic LP}

We had (49\%) of hypertrophic lichen planus. (Fig 9). It shows irregular hyperplasia of mainly follicular infundibular epithelium with focal hypergranulosis and a markedly thickened compact horny layer. The hyperplastic follicular infundibula show lichenoid infiltrates at their bases and sides with small subepidermal clefts..The interfollicular papillary dermis shows markedly thickened vertically oriented bundles of collagen, evidence of repeated rubbing of the lesion.

In Group IV-are cases of ID with psorisiform hyperplasia. Some of the conditions in this group are diseases which show interface changes as a secondary pathological feature and are therefore not classified as primary ID. True ID which show this pattern are LS and Lichenoid variant of persistent pigmented purpuric dermatitis.
In present study we had $50.41 \%$ cases with psoriariform hyperplasia .

We found $2.47 \%$ cases of LS.

Lichen Striatus: It is a combination of several patterns of inflammation, which may not be seen in individual lesions. Although classified as a primary interface dermatitis, it often shows psoriasiform hyperplasia of the epidermis with foci of mild to moderate spongiosis with few scattered individually necrotic keratinocytes in the spinous layers. Mounds of parakeratosis are often seen in the thickened stratum corneum. The interface changes in well-developed lesions may be minimal and a diagnosis of psoriasiform or spongiotic dermatitis may be given. Presence of a fairly dense peri-eccrine and periadnexal infiltrate of lymphocytes is the characteristic finding. Sometimes, this finding alone may serve to differentiate lichen striatus from other eczematous dermatoses ${ }^{[1,5,6]}$ In our study the LS showed(Fig 10) epidermis with psorisiform hyperplasia, dense infiltrate involving both superficial and deep dermis which obscures the basal layer of epidermis., spongiosis and the infiltrate involving the periadnexal and structures.

Grp IV-includes Cases of ID with Epidermal Atrophy: This represents the late atrophic phase of several dermatoses like atrophic LP ,LE and dermatomyositis ,acrodermatitis, chronic atrophicans, Lichen sclerosis et atrophicus, counter of old lesions of porokeratosis and poikiloderma due to various causes. In the present study we had $2.47 \%$ ) cases of epidermal atrophy. We found $3.30 \%$ cases of atrophic LP , $4.13 \%$ cases of SLE and 1.6 $\%$ cases of dermotomyositis. Sushma Desai et al found $2.5 \%$ cases of atrophic LP. Hegade et a ${ }^{[10]}$ noted ( $5.6 \%$ ) cases of atrophic LP in their studies.

Atrophic LP: Atrophic lesion may resemble parakeratosis clinically. Typical papules of LD are usually present at the margins A rare form of atrophic LP is composed of annular lesions. On histopathology: Epidermis is thin and there is loss of normal rete ridges pattern. The infiltrate is usually less dense than in typical $\mathrm{LP}^{(7)}$

Lupus Erythematosus (LE) It is an autoimmune disorder affecting skin, hematopoietic, and lymphoreticular organs, joints, kidney, lung, serosa, and cardiovascular structures in concert or in isolation. Lupus erythematosus is subdivided clinically into systemic (SLE), subacute cutaneous (SCLE), and discoid (DLE) forms, each with its own characteristic skin findings.

Lesions of SLE, for example, show a pauciinflammatoryinterface dermatitis with subtle basal layer vacuolopathy and no basement membrane zone thickening, keratotic follicular plugging, or acanthosis ${ }^{[1]}$ 
Dermatomyositis: Dermatomyositis combines an inflammatory myopathy with characteristic skin lesions: the often-subtle heliotrope rash, the Gottron papule, a violaceous or hypopigmented papule over the joints of the fingers, erythema of the up- per back (the "shawl sign"), extensive erythema of the extensor surfaces of the arms, scaly alopecia, and cuticular overgrowth with periungual telangiectasias. Skin lesions of dermatomyositis manifest an atrophying cell-poor lym- phocytic interface dermatitis accompanied by dermal mucinosis and vascular alterations that vary according to the age of the lesion biopsied and the presence or absence of myopathy: in patients with myopathic dermatomyositis, a characteristic injury pattern comprising a variably cell- poor, often thrombogenic lymphocytic vasculopathy mainly affecting the dermal papillae capillaries is seen ${ }^{[1,3]}$

In our study we had 02 cases of dermatomyositis with $100 \%$ clinical concordance.

The case of DM that we studied shows atrophic epidermis with cell poor lymphocytic IFD accompanied by mucinosis. (Fig.11)

\section{Conclusion}

A clinicopathologic correlation is absolutely essential for the final diagnosis of IFD. Presence of an interface lichenoid inflammatory reaction should not be the only criterion for the diagnosis of LP or any of its variants

\section{Acknowledgement}

The authors are thankful to the Department of Dermatology, Technicial Staff, BVDUMC \& H, Sangli, for their help and cooperation

\author{
Abbreviations used \\ IFD : Interface dermatitis \\ DE junction : Dermo epidermal junction \\ LP : Lichen Planus \\ ALP : Atrophic Lichen Planus \\ HLP : Hypertrophic Lichen Planus \\ EM : Erythema Multiforme \\ LE :Lupus Erythematosus \\ LDE : Lichenoid Drug Eruptions
}

PLC : Pityriasis Lichenoid Chronica

LSEA:Lichen sclerosiset atrophicus

GVHD : Graft Versus Host disease

LS : Lichen Striatus

DM : Dermatomyositis

PLEVA : Pityriasis Lichenoides et varioliform

TEN: Toxic Epidermal Necrolysis

\section{Reference}

1. Crowson AN, Magro CM, Mihm MC Jr. Interface Dermatitis. Arch Pathol Lab Med 2008 Apr; 132 (4) : 652-66.

2. Le Boit PE. Interface Dermatitis. How specific are its histopathologic features? Arch Dermatol 1993; 129:1324-28

3. Virendra Sehgal, G Srivastava et al. Lichenoid tissue reaction/Interface dermatitis: Recognition, classification, etiology \& clinicopathological overtones. Indian Journal of Dermatology, venerology and Leprology 2011; Vol 77: Issue 4:418-430.

4. Tilly JJ, Drolat BA, Esterley NB et al. Lichenoid eruptions in Children. J Am Acad Dermato;2004;51:606-24.

5. Rajiv Joshi. SYMPOSIUM DERMATOPATHOLOGY. Indian Journal of Dermatology, Venereology \& Leprology 2013;79:3: 349-59.

6. Philip Le boit. Dermatitis involving dermo-epidermal junction. In:Maize JC. Burgdorf WH, Hurt MA, Le Boit PE, Metcalf JS, Smith T et al, editors. Cutaneous Pathology. Philadelphia: Churchill Livingstone:1998.pg 87-145.

7. Weedon D. The Lichenoid Reaction Pattern. In: Weedon D, editor. Skin Pathology. 2 nd ed. New York: Churchill Livingstone; 2002. p. 31-74.

8. Sushama Desai, Anuya Badwe, Balkrishna Nikam et al. Histopathological study of Interface dermatitis with its clinical correlation. International journal of healthcare \& Biomedical research, Volume: 2, Issue: 3 , April 2014 , Pages 24-32

9. Suja Ajoy Kumar, G Nandakumar. A study of Interface Dermatitis with clinical correlation. Journal of Evolution Of Medical and Dental Sciences 2015 ; Vol . 4, Issue 42, May 25; Page: 7344-7351

10. Vijaya Kumar Hegde, Urmila $\mathrm{N}$ Khadilkar , A clinicopathological study of interface dermatitis. IOSR Journal of Dental and Medical Sciences (IOSR- JDMS) .2014. Volume 13, Issue 4 Ver. IV. (Apr. 2014), PP 105-107

*Corresponding author:

Dr. Vaibhav Mane, Flat No. 1 Shri Ramshailya Aparrtment, Neminathnagar, Sangli 416415. INDIA

Phone: +91 9422041490

Email: vaishnavilab1060@gmail.com

Financial or other Competing Interests: None.

Date of Submission : 05.04.2017

Date of Acceptance : 18.04.2017

Date of Publication : 21.04.2017 